\title{
New immune regulation strategy in the age of regenerative medicine using pluripotent stem cells
}

\author{
Haruka Wada', Hiroya Kudo ${ }^{1,2)}$, Hajime Sasaki ${ }^{1,3)}$, \\ Muhammad Baghdadi" and Ken-ichiro Seino ${ }^{1, *}$ \\ ${ }^{1)}$ Institute for Genetic Medicine, Hokkaido University, Sapporo, Japan \\ ${ }^{2}$ Department of Urology St. Marianna University School of Medicine, Kawasaki, Kanagawa, Japan \\ ${ }^{3)}$ Department of Renal and Genitourinary Surgery, Graduate school of medicine, Hokkaido University, Sapporo, Japan
}

Recent progress of manipulating pluripotent stem cells expands possibilities of regenerative medicine and opens novel transplantation medicine. However, in many cases of these medicines, the relationship between therapeutic cells and recipients would be allogeneic. In this context, we proposed new concept of immune regulation therapy in new-age medicine using pluripotent stem cells. In our concept, not only grafts but also immune regulating cells are generated from pluripotent stem cells by exertion of its pluripotency. We have recently developed immune suppressive macrophage-like cells from pluripotent stem cells. These cells suppressed allo-antigen stimulated $T$ cell proliferation in an iNOS dependent manner. Furthermore, these immune suppressive macrophage-like cells derived from pluripotent stem cells prolonged survival of grafts derived from same pluripotent stem cells in allogeneic recipients. Thus, series of our study proved the efficacy of our new immune regulating strategy in the age of regenerative medicine which utilize pluripotent stem cells as a therapeutic cell source.

Rec.8/6/2015, Acc.10/18/2015, pp238-243

${ }^{*}$ Correspondence should be addressed to:

Ken-ichiro Seino, MD, PhD, Division of Immunobiology, Institute for Genetic Medicine, Hokkaido University, Kita-15, Nishi-7, Sapporo 060-0815, Japan. Phone: +81-11-706-5532, Fax: +81-11-706-7545, E-mail: seino@igm.hokudai.ac.jp

Key words ES cells, iPS cells, transplantation, regenerative medicine, immune regulation

\section{Introduction}

Cell transplantation using pluripotent stem cells as a source has been developed remarkably in recent years. It is still difficult to make well-structured three dimensional organs in vitro from pluripotent stem cells so far, but there are already many reports that succeeded to make many kinds of tissues or functional cells from pluripotent stem cells (Reviewed in Ref.1). Therefore, cell therapy or regenerative medicine using pluripotent stem cells is highly anticipated as reality. In 2014, retinal pigment epithelium cells generated 
from human iPS cells were transplanted into a patient suffering from age-related macular degeneration (Nature doi: 10.1038/nature.2014,15915). This case, autologous iPS cells were used as the cell source. However, making iPS cells for individual patient is impractical because it needs huge cost and much time before yielding safety-guaranteed ones. To overcome this point, iPS cell banking project is in progress $^{2)}$.

\section{Pluripotent stem cells and transplantation medicine}

One of the big advantages using iPS cells is that one could expect an achievement of rejection-free transplantation when using autologous iPS cells. In the iPS cell banking project, original cells were collected from donors who bear homogeneous HLA allele to reduce immunological rejection. However, the immunological concerns still remain because many kinds of proteins in allogeneic cells can be possible antigens. In these days, there are many superior immune suppressive drugs against immune rejection ${ }^{3)}$. So, it is common to use these drugs to avoid and reduce the allogeneic rejection. But long-term use of such drugs sometimes causes severe side effects, e.g., development of a malignant neoplasm and opportunistic infection ${ }^{4)}$. In this context, immune suppressive therapies without the use of immunosuppressants are strongly expected.

\section{Novel immune regulating strategy for new-age transplantation medicine using pluripotent stem cells}

Pluripotent stem cells, including ES cells ${ }^{5,6)}$ and iPS cells ${ }^{7,8)}$, have a potential to differentiate into many kinds of cell-types. Immune cells, including immune regulatory cells, are one of them. So, such immune regulatory cells have a possibility to suppress immune rejection of allogeneic grafts. We have proposed a new immune regulation strategy in the age of regenerative medicine using pluripotent stem cells $^{9,10)}$ (Fig. 1A). In this strategy, pluripotent stem cells differentiate into grafts as well as immune regulatory cells. On the occasion of allogeneic transplantation, treatment with the immune regulatory cells may reduce the risk of immune rejection and prolong the graft survival.

To realize our concept, a mouse transplantation model was developed in which both grafts and immune regulatory cells were derived from pluripotent stem cells (Fig. 2). We used 129/SvJ mice-derived ES cells (E14, haplotype b) as a source of pluripotent stem cells (graft donor) and $\mathrm{C} 3 \mathrm{H}$ mice

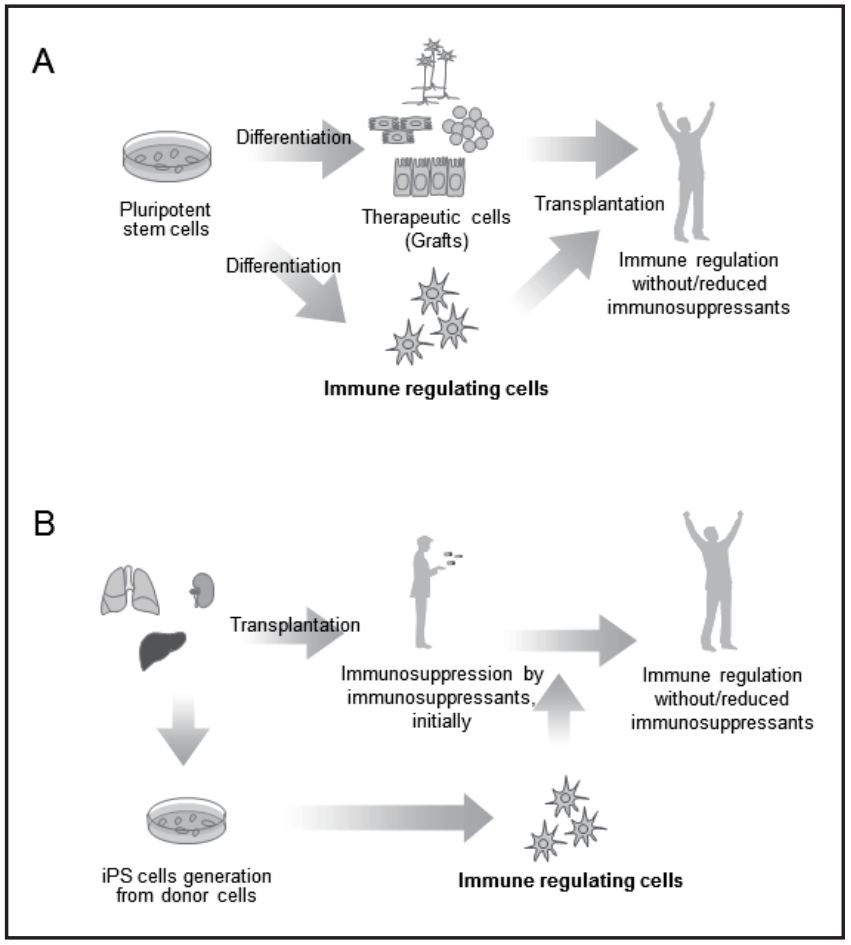

Fig. 1 (A) A new immune regulation strategy in the age of regenerative medicine using pluripotent stem cells.

(B) New immune regulation strategy using pluripotent stem cells technology in the current transplantation medicine.

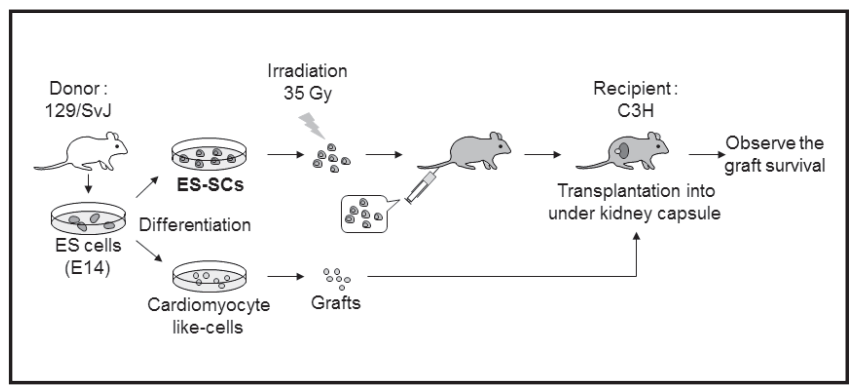

Fig. 2 Experimental design of new immune regulation strategy in the age of regenerative medicine using pluripotent stem cells.

(haplotype k) as a recipient. This ES cells and $\mathrm{C} 3 \mathrm{H}$ mice are allogeneic each other. E14 ES cells were differentiated into cardiomyocytes ${ }^{11)}$, which were transplanted as grafts, and also into immune regulatory macrophage-like cells ${ }^{9,10)}$. 


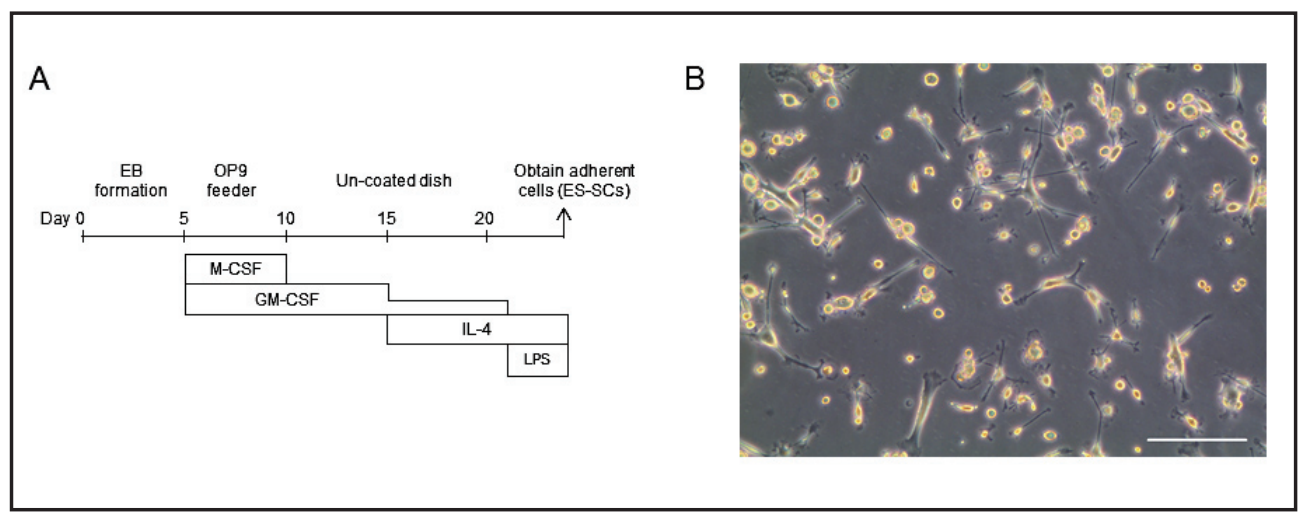

Fig. 3 (A) Overall strategy for generating immune-suppressive macrophages from pluripotent stem cells.

(B) Morphology of ES-SCs. Bar: $100 \mu \mathrm{m}$

\section{Induction of macrophage-like immune suppressive cells from pluripotent stem cells}

There are three general steps in culture protocol to obtain immune suppressive cells from pluripotent stem cells. The first step is a differentiation into mesoderm cells that can become hematopoietic lineage cells. The second step is an induction of hematopoietic cells and directed-differentiation to macrophage-like cells. The last step is conferring immune suppressive capacity to the generated macrophage-like cells. The outline of protocol to generate macrophage like immune suppressive cells form pluripotent stem cells ${ }^{9,10}$ was illustrated in Figure 3A.

Withdrawal of Leukemia inhibitory factor (LIF) from culture media, pluripotent stem cells start differentiation ${ }^{12)}$. LIF is one of the key molecules to maintain pluripotency in pluripotent stem cells. On day around 5 of the suspension culture in LIF free media, sphere-like embryoid body formation was observed. Embryoid body usually contains all three germ layer cells, endoderm, mesoderm and ectoderm cells. After the dissociation of embryoid bodies including Flk $-1^{+}$mesoderm cells into single suspension by trypsin treatment, those cells were plated on OP9 stromal cells. OP9 stromal cells are frequently used to support hematopoietic cell differentiation ${ }^{13)}$. And then, we added some cytokines including Csf1 (M-CSF) or Csf2 (GM-CSF) to support macrophage differentiation. On day around 10 , loosely attached hematopoietic cells to OP9 stromal cells could be observed. Those hematopoietic cells were harvested, and then cultured on non-treated culture dishes in the existence of Csf2. Thereafter, IL-4 was added on day around 15. On day around day 20 , dendritic cell-like floating sphere could be obtained. 48 hours after adding lipopolysaccharide, irregularly shaped macrophage-like cells which tightly adhered to non-treated culture dish could be observed (Fig. 3B). Subsequent analysis revealed that these macrophage-like cells have strong immune suppressive function $^{\text {9) }}$, so we called these cells ES (or iPS)SCs (ES cells (or iPS cells) derived immune suppressive cells). We have also observed that similar immune suppressive cells could be induced from human iPS cells ${ }^{10)}$.

\section{Features of macrophage-like immuno- suppressive cells derived from pluripotent stem cells \\ Detailed analysis by flow cytometry revealed that both ES-} SCs and iPS-SCs have macrophage features supported by highly expression of CD45 (a hematopoietic cell marker), and CD11b and F4/80 (macrophage cell markers) ${ }^{9,10)}$. Gene expression analysis showed that some kinds of immunesuppressive molecules such as Arg1 (Agrinase 1) and Nos2 (Nitric oxide synthase 2, encoding iNOS) were expressed in both ES-SCs and iPS-SCs ${ }^{9,10}$. These results suggested that these cells have immune suppressive function. In microarray analysis, ES-SCs were compared to ES cell derived dendritic cells (ES-DCs $)^{9)}$. Gene expression correlated with T cell suppression was higher in ES-SCs than ES-DCs ${ }^{9}$. On the other hand, genes related to $T$ cell stimulation, such as antigen presenting molecules were expressed relatively higher in ES-DCs than in ES-SCs ${ }^{9}$. These series of results generally suggested that ES- and iPS-SCs might act as immune suppressive cells. 
Macrophage-like immune suppressive cells suppress alloantigen-activated $\mathrm{T}$ cell proliferation

Since $\mathrm{T}$ cells play important roles in graft rejection in allogeneic transplantation ${ }^{14)}$, its regulation is quite important for graft survival. Therefore, we tested immune suppressive function of ES- and iPS-SCs against allo-antigen stimulated $\mathrm{T}$ cell proliferation. We prepared dendritic cells as antigen presenting cells from bone marrow of allogeneic mice. $T$ cells efficiently proliferated when co-cultured with the allogeneic dendritic cells. However, in addition of ESor iPS-SCs into the co-culture, T cell proliferation was significantly inhibited $^{9,10)}$. Further analysis revealed that this inhibitory function of ES- and iPS-SCs was supported in an iNOS dependent manner ${ }^{9,10)}$.

\section{Pluripotent stem cells-derived immune suppressive cells prolonged survival of allogeneic grafts}

Furthermore, immune suppressive function of ES- and iPS-SCs was tested in vivo allogeneic transplantation model (Fig. 2). To perform this, we prepared cardiomyocytes or embryoid bodies as grafts from the same ES- or iPS-cells with ES- or iPS-SCs' source.

In in vivo model, irradiated ES-SCs were administered into allogeneic recipients before the graft transplantation. Compared with mice without the ES-SCs treatment, it significantly prolonged the graft survival ${ }^{9)}$. Importantly, ex vivo analysis revealed that formation of donor-type specific immune suppression in recipients was observed ${ }^{9)}$. Briefly, splenic $T$ cells were harvested from ES-SCs treated recipient mice. Then, $T$ cell proliferation assay by the stimulation of dendritic cells from syngeneic, allogeneic but same background as ES-SCs or 3rd party mice was performed. T cells from ES-SCs-treated mice proliferated minimally by stimulation with DCs of syngeneic as well as allogeneic but same background as ES-SCs. On the other hand, T cells from ES-SCs-treated mice significantly proliferated when stimulated with DCs of 3rd party mice. These results suggest that ES-SCs induce immune suppression in a donor antigen specific manner" ${ }^{9}$. Pathological analysis showed that reduced $\mathrm{T}$ cell infiltration in the grafts was observed in ES-SCs treated mice ${ }^{9)}$. Interestingly, the administered ES-SCs were not observed in the transplanted site $^{9}$. This result suggested that immune suppressive function of ES-SCs was exerted systemically rather than locally. Similar results were generally obtained when iPS-SCs were used as immune suppressive cells in in vivo experiments ${ }^{10)}$.

\section{Administration of the immune suppres- sive cells into allogeneic recipients suppressed anti-allo antigen antibody production in vivo}

We further analyzed immune suppressive function of these immune suppressive cells in vivo ${ }^{10)}$. Allogeneic grafts were transplanted into recipients with or without iPS-SCs treatment. Then we evaluated immune suppressive potential of iPS-SCs in vivo by monitoring antibody production against allogeneic antigen. Interestingly, allo-antibody production was significantly suppressed in iPS-SCs-treated recipients ${ }^{10}$. Thus, iPS-SCs could really exert immune suppressive function in vivo, at least partly, by suppressing anti-alloantigen antibody production.

The immune regulation strategy using pluripotent stem cells could be applicable for current transplantation medicine

The series of our experiments proved the potential of our concept of immune suppressive therapy in cell transplantation using pluripotent stem cells. That is to generate not only grafts but also immune suppressive cells from the same pluripotent stem cells and then to subject to cell transplantation. Importantly, our concept could be applicable for the current allogeneic organ transplantation (Fig. 1B). Briefly, one can generate iPS cells from a piece of grafts, and then differentiate them into the immune suppressive cells. Administration of these immune suppressive cells may inhibit allogeneic rejection, and reduce or cease the need of immunosuppressants.

\section{Directions for future research and de- velopment}

In this review, we have introduced macrophage-like immune suppressive cells derived from pluripotent stem cells, which could prolong allogeneic graft survival. However there are some issues remaining. The major one is its limited duration of immune suppressive function. The life-long graft survival is ultimate goal. Some kinds of cells which have potential to induce life-long tolerance have been suggested. Hematopoietic stem cells ${ }^{15,16)}$ are a good example. Generation of hematopoietic stem cells from pluripotent stem cells have already reported ${ }^{17)}$. Actually, it has been reported that hematopoietic chimerism by ES- 
cell-derived hematopoietic stem cells allowed donor type cardiac allo-graft survival ${ }^{17)}$. However, meanwhile, some oncogenes are required to generate hematopoietic stem cells from pluripotent stem cells. Thus, there are several problems remained to be solved, but the strategy could be a breakthrough of immune regulation in the age of cell transplantation using pluripotent stem cells.

In the not-so-distant future, increasing use of pluripotent stem cells as source of cells/tissues for regenerative medicine is anticipated. In many cases, the relationship between cells/tissues derived from pluripotent stem cells and recipients would be allogeneic. Importantly, all cases are allogeneic in the use of ES cells. Therefore, some immune manipulation should be considered. Our recent studies have shown the possibility of new concept of immune regulation, which could be applied to new-age transplantation medicine. In brief, in the new-age medicine which uses pluripotent stem cells, one can generate both therapeutic cells and immune-regulating cells from the same pluripotent stem cells. By administrating the immune regulating cells, protection of grafts from immune rejection, or reduction or cease of immunosuppressants are expected. We believe that the strategy generating immune regulatory cells from pluripotent stem cells would be more important than expected so far.

\section{Acknowledgement and Source of funding}

The authors thank Mr. Hyuma Tsuji, Mr. Ryo Otsuka and all the members of our laboratory. This study was supported in part by research grants from the Ministry of Education, Culture, Sports, Science and Technology of Japan to HW and KS. This work was also supported in part by the Uehara Memorial Foundation to KS.

\section{Conflict of interests}

The funders had no role in study design, data collection and analysis, decision to publish, or preparation of the manuscript.

\section{References}

1) Tabar V, Studer L: Pluripotent stem cells in regenerative medicine: challenges and recent progress. Nat Rev Genet. 2014; 15: 82-92.

2) Nakatsuji N, Nakajima F, Tokunaga K: HLA-haplotype banking and iPS cells. Nat Biotechnol. 2008; 26: 739-740.

3) Halloran PF: Immunosuppressive drugs for kidney transplantation. N Engl J Med. 2004; 351: 2715-2729.

4) Sayegh $\mathrm{MH}$, Carpenter CB: Transplantation 50 years later--progress, challenges, and promises. N Engl J Med. 2004; 351: 2761-2766.

5) Evans MJ, Kaufman MH: Establishment in culture of pluripotential cells from mouse embryos. Nature. 1981; 292: 154-156.

6) Thomson JA, Itskovitz-Eldor J, Shapiro SS, Waknitz MA, Swiergiel JJ, Marshall VS, et al: Embryonic stem cell lines derived from human blastocysts. Science. 1998; 282: 1145-1147.

7) Takahashi K, Yamanaka S: Induction of pluripotent stem cells from mouse embryonic and adult fibroblast cultures by defined factors. Cell. 2006; 126: 663-676.

8) Takahashi K, Tanabe K, Ohnuki M, Narita M, Ichisaka T, Tomoda K, et al: Induction of pluripotent stem cells from adult human fibroblasts by defined factors. Cell. 2007; 131: 861-872.

9) Kudo $H$, Wada $H$, Sasaki $H$, Tsuji $H$, Otsuka R, Baghdadi $\mathrm{M}$, et al: Induction of Macrophage-Like Immunosuppressive Cells from Mouse ES Cells That Contribute to Prolong Allogeneic Graft Survival. PLoS One. 2014; 9: e111826.

10)Sasaki $H$, Wada $H$, Baghdadi $M$, Tsuji $H$, Otsuka $R$, Morita K, et al: New immunosuppressive cell therapy to prolong survival of induced pluripotent stem cell-derived allografts. Transplantation. 2015; in press.

11) Yuasa $S$, Itabashi $Y$, Koshimizu U, Tanaka T, Sugimura $K$, Kinoshita M, et al: Transient inhibition of BMP signaling by Noggin induces cardiomyocyte differentiation of mouse embryonic stem cells. Nat Biotechnol. 2005; 23: 607-611.

12)Williams RL, Hilton DJ, Pease S, Willson TA, Stewart $\mathrm{CL}$, Gearing DP, et al: Myeloid leukaemia inhibitory factor maintains the developmental potential of embryonic stem cells. Nature. 1988; 336: 684-687.

13)Kitajima K, Tanaka M, Zheng J, Sakai-Ogawa E, Nakano T: In vitro differentiation of mouse embryonic stem cells to hematopoietic cells on an OP9 stromal cell monolayer. Methods Enzymol. 2003; 365: 72-83.

14)Turka LA, Linsley PS, Lin H, Brady W, Leiden JM, Wei $R Q$, et al: T-cell activation by the CD28 ligand $B 7$ is required for cardiac allograft rejection in vivo. Proc Natl Acad Sci U S A. 1992; 89: 11102-11105.

15) Ildstad ST, Sachs DH: Reconstitution with syngeneic plus allogeneic or xenogeneic bone marrow leads to specific acceptance of allografts or xenografts. Nature. 1984; 307: 168-170.

16)Kawai T, Cosimi AB, Spitzer TR, Tolkoff-Rubin N, 
Special Issue (Mini Review) New immune regulating strategy using PSCs

Inflammation and Regeneration Vol.35 No.5 November 2015

Suthanthiran M, Saidman SL, et al: HLA-mismatched renal transplantation without maintenance immunosuppression. N Engl J Med. 2008; 358: 353-361.
17)Bonde S, Chan KM, Zavazava N: ES-cell derived hematopoietic cells induce transplantation tolerance. PLoS One. 2008; 3: e3212. 